\title{
Diet and airway inflammation
}

There has been a recent explosion of data on the mechanisms by which diet influences inflammatory diseases. Two studies published in Nature Medicine detail distinct molecular mechanisms by which diet can influence airway inflammation.

Asthma is a heterogeneous disease that is driven by distinct immunological pathways. Recent studies have shown that obesity is a major risk factor for the development of non-allergic asthma. In their study, Kim et al. investigated the molecular mechanisms of obesity-associated asthma. Mice fed a high-fat diet for 14 weeks developed obesity and also airway hyperreactivity (AHR), a cardinal feature of asthma. The authors found that this was associated with production of the pro-inflammatory cytokine interleukin-17A (IL-17A). Indeed, IL-17A levels were greatly increased in the lungs of mice fed a high-fat diet, and $1117 a^{-1-}$ mice on a high-fat diet did not develop AHR. Interestingly, the main source of IL-17A in the lungs of obese mice was not $\mathrm{T}$ helper $17\left(\mathrm{~T}_{\mathrm{H}} 17\right)$ cells, as mice lacking B and T cells (Rag1 $1^{-/-}$mice) still developed AHR when given a high-fat diet. Instead, the numbers of type 3 innate lymphoid cells (ILC3s) in the lungs of obese mice were greatly increased, and these cells were found to be the main producers of IL-17A. Indeed, ILC3s could mediate AHR when transferred to immunocompromised mice ( $\mathrm{Rag}^{-/-} \mathrm{Il}_{2 \mathrm{rg}^{-1}}$ mice) on a normal diet treated with recombinant IL-1 $\beta$.

Obese mice also had increased numbers of lung inflammatory macrophages, and these cells expressed IL- $1 \beta$, which is a known inducer of IL-17A production by

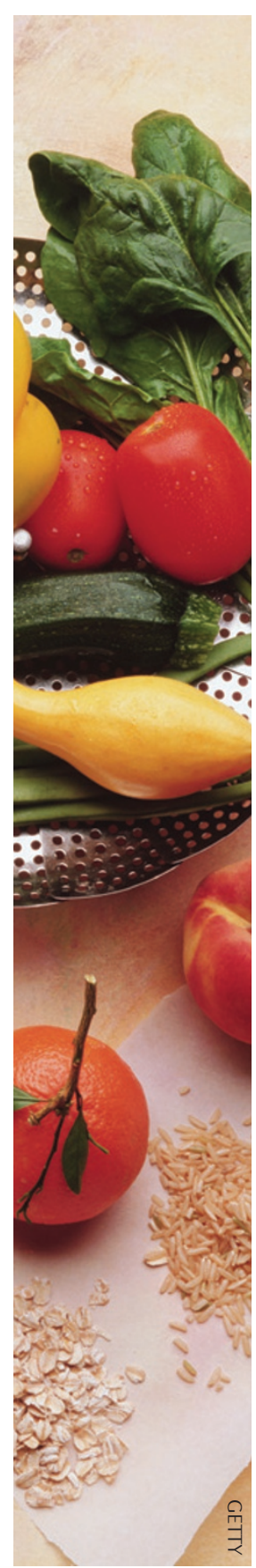

$\mathrm{T}_{\mathrm{H}} 17$ cells and ILC3s. The production of IL- $1 \beta$ by lung macrophages was dependent on the NOD-, LRRand pyrin domain-containing 3 (NLRP3) inflammasome, and $N \operatorname{lrp} 3^{-1-}$ mice on a high-fat diet failed to develop AHR. Furthermore, blocking IL- $1 \beta$ signalling with the IL- $1 \beta$ receptor antagonist anakinra in obese mice abolished AHR and reduced the number of lung ILC3s.

These data suggest that AHR in obese mice is associated with the activation of the NLRP3 inflammasome, the production of IL- $1 \beta$ and increased numbers of IL-17Aproducing ILC3s in the lungs. Finally, the authors showed that patients with severe asthma have higher numbers of IL-17A $\mathrm{A}^{+}$ILC3-like cells in bronchoalveolar lavage fluid compared with individuals with mild asthma or no asthma.

Trompette et al. investigated the potential beneficial effects of dietary fibre on allergic lung inflammation. They found that when mice fed a high-fibre diet were exposed to house dust mite extract (HDM), they developed less severe allergic airway inflammation and showed reduced AHR compared with mice on a control diet. In addition, dendritic cells (DCs) isolated from the lungs of mice on a high-fibre diet showed a less-activated phenotype than DCs from mice on the control diet.

But how does dietary fibre affect disease severity and the activation state of DCs in the lung? An analysis of the gut and lung microbial communities from mice fed diets of varying fibre content showed that high fibre intake increased the proportion of Bacteroidaceae and
Bifidobacteriaceae in the microbiota, whereas a low-fibre diet led to a microbiota that was dominated by Firmicutes. Bacteroidaceae ferment fibre into short-chain fatty acids (SCFAs), including propionate, and an increase in SCFA levels in the caecum and serum was observed in mice on the high-fibre diet. Treatment of mice with propionate reduced the lung inflammatory response from day 2 after the final HDM exposure, and this effect of propionate was dependent on the SCFA receptor G protein-coupled receptor 41 (GPR41; also known as FFAR3).

However, SCFAs were not detectable in the lungs, so the authors assessed whether SCFA treatment influences newly recruited lung DCs. Indeed, they found that propionate treatment enhanced the numbers of DC precursors in the bone marrow. Furthermore, the DCs seeding the lungs in response to HDM challenge in propionatetreated mice had characteristics of immature DCs and were ineffective at driving $\mathrm{T}_{\mathrm{H}} 2$ cell responses. Thus, the allergic airway inflammatory response could not be sustained in propionate-treated mice.

Together, these studies shed light on how diet can influence lung inflammation and highlight specific immunological pathways that might be targeted to treat distinct forms of lung inflammation.

Olive Leavy

ORIGINAL RESEARCH PAPERS Kim, H. Y. et al. Interleukin-17-producing innate lymphoid cells and the NLRP3 inflammasome facilitate obesityassociated airway hyperreactivity. Nature Med. 20 54-61 (2014)| Trompette, A. et al. Gut microbiota metabolism of dietary fiber influences allergic airway disease and hematopoiesis. Nature Med. http://dx.doi.org/10.1038/nm.3444 (2014) 